\title{
Comparison of cone beam computed tomography and intra oral periapical radiography in detecting periapical lesions $-\mathrm{A}$ systematic review
}

\author{
DR.M Priyanka ${ }^{1}$ B.D.S.,DR.Sujeer ${ }^{2}$ M.D.S.,DR.Toby Thomas ${ }^{3}$ M.D.S., \\ 1-Student,Dept.of conservative and endodontics, Saveetha dental college, Chennai,Tamil Nadu,India, \\ 2-Professor, Dept.of conservative and endodontics, Saveetha dental college,Chennai,Tamil Nadu,India, \\ 3- Assistant professor, Dept.of conservative and endodontics, Saveetha dental college, Chennai,Tamil \\ Nadu,India
}

\begin{abstract}
:
Introduction: Cone-beam computerized tomography (CBCT) is a medical image acquisition technique based on a cone-shaped X-ray beam centered on a two-dimensional (2D) detector. The source-detector system performs one rotation around the object producing a series of $2 D$ images. The images are reconstructed in a three-dimensional (3D) data set using a modification of the original cone-beam algorithm developed by Feldkamp ${ }^{1}$ et al in 1984. This technique is widely used in different industrial and biomedical applications such as micro- CT. Among the first clinical applications were single photon emission computerized tomography (SPECT), angiography and image-guided radiotherapy. Dedicated cone-beam computerized tomography scanners for the oral and maxillofacial (OMF) region were pioneered in the late 1990s independently by Arai ${ }^{2}$ et al. in Japan.

This Systematic review aims at comparing cone-beam computerized tomography and periapical radiography in detection of periapical lesion.

Objective: To compare the accuracy of cone beam computed tomography (CBCT) and intra oral periapical radiography (IOPA) in diagnosis of periapical pathology

Search Strategy: Database such as Pub med Central and Medline were searched for the related topics from February 1996 till July 2013.

Selection Criteria: Trials were selected if they met the following criteria: Clinical trials comparing the accuracy of Cone beam computed tomography (CBCT) and intra oral periapical radiography (IOPA).

Data Collection and Analysis: All the studies included were based on the data extraction and analysis of the studies for quality and publication bias. The data collection form was customized. The primary outcome is to compare Cone beam computed tomography (CBCT) and intra oral periapical radiography (IOPA) in detection of periapical lesions.

Main Results: The review concluded that cone beam computed tomography is superior to conventional intra oral periapical radiography in detection of periapical lesions.

Conclusion: The probability of detecting periapical lesions was more in case of cone-beam computerized tomography when compared to conventional intra oral periapical radiography where external factors such as anatomical noise and poor irradiation geometry which are not in operators control are eliminated.
\end{abstract}

\section{Background}

Traditionally, the diagnosis of periapical lesion was based on clinical and radiographic presentations; confirmatory diagnosis was only by a biopsy which was highly impossible in the case of a non surgical procedure. If biopsy is taken the treatment is no longer a non surgical procedure (Simon et al 1980) ${ }^{3}$. Intra oral periapical radiograph could determine only the mesiodistal aspect of the periapical lesion (Kaffe et al 1988) ${ }^{4}$ since it is a two dimensional representation of a three dimensional object. Periapical lesions confined within cancellous bone were not usually detected in periapical radiography. It has been reported that CBCT scans detected periapical lesions in many cases which is absent in periapical radiograph ${ }^{5,6,7}$. Moreover CBCT helps in detection of extent of lesion not only mesiodistally but also buccolingually

AIM

The aim of this systematic review was to compare the accuracy of cone beam computed tomography $(\mathrm{CBCT})$ and intra oral periapical radiography (IOPA) in the diagnosis of periapical lesions.

\section{Structured Questions}


Is there any difference in detecting periapical radiolucency between Cone beam computed tomography and intra oral periapical radiography in detection of periapical lesion?

\section{Pico Analysis}

- Population- Patients with periapical pathology.

- Intervention- Cone beam computed tomography

- Comparison- Intra oral periapical radiography.

- Outcome- Detection of periapical lesion.

\section{Null Hypothesis}

There is no difference in detection between Cone beam computed tomography and intra oral periapical radiography.

\section{Materials And Methods}

\section{Sources Used}

For identification of studies included and considered in this review, a detailed search strategy was developed for the database searched. The MEDLINE search used the combination of controlled vocabulary and free text terms.

\section{Searched Databases}

- PUBMED ( From February 1996 till July 2013)

- PUBMED Advanced Search (From February 1996 till July 2013)

- MEDLINE

\section{Language}

No language restrictions

\section{Hand Searching}

All issues of the following journals were hand searched as being of particular importance to the review.

- Journal of Endodontics

- International Endodontic Journal

- Journal of American Dental Association

- Journal of Dentistry

- Oral Medicine, Oral Pathology, Oral Surgery, Oral Radiology and Endodontics.

- British Dental Journal

- Endodontic Topics

\section{History}

III. Search Methodology

Download historyClear history

Recent queries

\begin{tabular}{|c|c|c|c|c|}
\hline Search & $\begin{array}{l}\text { Add to } \\
\text { builder }\end{array}$ & Query & $\begin{array}{l}\text { Items } \\
\text { found }\end{array}$ & Time \\
\hline$\underline{\# 40}$ & $\underline{\text { Add }}$ & $\begin{array}{l}\text { Search }(((()((()((()((\text { periapical lesion) OR periapical abscess) OR periapical granuloma) } \\
\text { OR periapical cyst) OR chronic apical periodontitis) OR necrotic teeth) OR non vital } \\
\text { teeth) OR periapical pathology) OR periapical pathosis) OR periapical periodontitis) OR } \\
\text { acute apical abscess) OR chronic apical abscess) OR periradicular pathosis) OR } \\
\text { periradicular abscess)) AND (((cone beam computed tomography) OR CBCT) OR } \\
\text { computed tomography) OR computerized tomography)) AND ((()(((conventional } \\
\text { radiograph) OR intra oral periapical radiograph) OR intra oral periapical radiography) } \\
\text { OR conventional radiography) OR intra oral radiography) OR intra oral radiograph) OR } \\
\text { periapical radiograph) OR periapical radiography)) AND (((()(((accuracy) OR } \\
\text { comparison) OR evaluation) OR detection) OR diagnosis) OR interpretation) OR } \\
\text { observation) OR management) OR differential diagnosis) }\end{array}$ & $\underline{224}$ & $04: 23: 20$ \\
\hline$\# 39$ & $\underline{\text { Add }}$ & $\begin{array}{l}\text { Search }(((((((\text { accuracy) OR comparison) } O R \text { evaluation) OR detection) OR diagnosis) OR } \\
\text { interpretation) OR observation) OR management) OR differential diagnosis }\end{array}$ & $\underline{10756852}$ & $04: 22: 50$ \\
\hline$\# 38$ & $\underline{\text { Add }}$ & Search differential diagnosis & $\underline{4575194}$ & $04: 21: 32$ \\
\hline$\# 37$ & $\underline{\text { Add }}$ & Search management & $\underline{1785522}$ & $04: 21: 14$ \\
\hline$\underline{\# 36}$ & $\underline{\text { Add }}$ & Search observation & $\underline{217319}$ & $04: 20: 59$ \\
\hline \#35 & $\underline{\text { Add }}$ & Search interpretation & $\underline{216716}$ & $04: 20: 48$ \\
\hline
\end{tabular}




\begin{tabular}{|c|c|c|c|c|}
\hline \multicolumn{5}{|c|}{ Recent queries } \\
\hline Search & $\begin{array}{l}\text { Add } \\
\text { builder }\end{array}$ & to Query & $\begin{array}{l}\text { Items } \\
\text { found }\end{array}$ & Time \\
\hline$\# 34$ & $\underline{\text { Add }}$ & Search diagnosis & $\underline{8158246}$ & 04:20:36 \\
\hline$\# 33$ & $\underline{\text { Add }}$ & Search detection & $\underline{577312}$ & 04:20:24 \\
\hline$\# 32$ & $\underline{\text { Add }}$ & Search evaluation & $\underline{1765554}$ & 04:20:09 \\
\hline$\# 31$ & $\underline{\text { Add }}$ & Search comparison & $\underline{709921}$ & 04:19:56 \\
\hline$\# 30$ & $\underline{\text { Add }}$ & Search accuracy & $\underline{213795}$ & 04:19:45 \\
\hline$\# 29$ & $\underline{\text { Add }}$ & $\begin{array}{l}\text { Search (((cone beam computed tomography) OR CBCT) OR computed tomography) OR } \\
\text { computerized tomography }\end{array}$ & $\underline{383079}$ & 04:18:56 \\
\hline$\# 28$ & $\underline{\text { Add }}$ & Search computerized tomography & $\underline{295807}$ & 04:18:25 \\
\hline$\# 27$ & $\underline{\text { Add }}$ & Search computed tomography & $\underline{376114}$ & 04:18:09 \\
\hline$\# 26$ & $\underline{\text { Add }}$ & Search CBCT & $\underline{2211}$ & 04:17:57 \\
\hline$\# 25$ & $\underline{\text { Add }}$ & Search cone beam computed tomography & $\underline{4239}$ & 04:17:45 \\
\hline$\# 24$ & $\underline{\text { Add }}$ & $\begin{array}{l}\text { Search (((c((conventional radiograph) OR intra oral periapical radiograph) OR intra oral } \\
\text { periapical radiography) OR conventional radiography) OR intra oral radiography) OR } \\
\text { intra oral radiograph) OR periapical radiograph) OR periapical radiography }\end{array}$ & $\underline{30194}$ & 04:17:03 \\
\hline$\# 23$ & $\underline{\text { Add }}$ & Search periapical radiography & $\underline{2450}$ & 04:16:20 \\
\hline$\# 22$ & $\underline{\text { Add }}$ & Search periapical radiograph & $\underline{319}$ & 04:16:01 \\
\hline$\# 21$ & $\underline{\text { Add }}$ & Search intra oral radiograph & $\underline{97}$ & 04:15:40 \\
\hline$\# 20$ & $\underline{\text { Add }}$ & Search intra oral radiography & $\underline{1064}$ & $04: 15: 21$ \\
\hline$\# 19$ & $\underline{\text { Add }}$ & Search conventional radiography & $\underline{26849}$ & 04:14:55 \\
\hline$\# 18$ & $\underline{\text { Add }}$ & Search intra oral periapical radiography & $\underline{87}$ & 04:14:25 \\
\hline$\# 17$ & $\underline{\text { Add }}$ & Search intra oral periapical radiograph & $\underline{25}$ & 04:14:17 \\
\hline$\# 16$ & $\underline{\text { Add }}$ & Search conventional radiograph & $\underline{743}$ & 04:13:59 \\
\hline$\# 15$ & $\underline{\text { Add }}$ & $\begin{array}{l}\text { Search }(((()(((()((\text { periapical lesion) OR periapical abscess) OR periapical granuloma) OR } \\
\text { periapical cyst) OR chronic apical periodontitis) OR necrotic teeth) OR non vital teeth) } \\
\text { OR periapical pathology) OR periapical pathosis) OR periapical periodontitis) OR acute } \\
\text { apical abscess) OR chronic apical abscess) OR periradicular pathosis) OR periradicular } \\
\text { abscess }\end{array}$ & $\underline{9867}$ & 04:13:20 \\
\hline$\# 14$ & $\underline{\text { Add }}$ & Search periradicular abscess & $\underline{71}$ & 04:12:07 \\
\hline$\# 13$ & $\underline{\text { Add }}$ & Search periradicular pathosis & $\underline{49}$ & 04:11:55 \\
\hline$\# 12$ & $\underline{\text { Add }}$ & Search chronic apical abscess & $\underline{197}$ & 04:11:43 \\
\hline$\# 11$ & $\underline{\text { Add }}$ & Search acute apical abscess & $\underline{261}$ & 04:11:31 \\
\hline$\underline{\# 10}$ & $\underline{\text { Add }}$ & Search periapical periodontitis & $\underline{4179}$ & 04:11:11 \\
\hline$\# 9$ & $\underline{\text { Add }}$ & Search periapical pathosis & $\underline{226}$ & 04:10:45 \\
\hline$\underline{\# 8}$ & $\underline{\text { Add }}$ & Search periapical pathology & $\underline{2057}$ & 04:10:07 \\
\hline$\# 7$ & $\underline{\text { Add }}$ & Search non vital teeth & $\underline{750}$ & 04:09:54 \\
\hline$\underline{\# 6}$ & $\underline{\text { Add }}$ & Search necrotic teeth & $\underline{2997}$ & 04:09:32 \\
\hline$\underline{\# 5}$ & $\underline{\text { Add }}$ & Search chronic apical periodontitis & $\underline{738}$ & 04:09:18 \\
\hline$\# 4$ & $\underline{\text { Add }}$ & Search periapical cyst & $\underline{1525}$ & 04:08:58 \\
\hline$\underline{\# 3}$ & $\underline{\text { Add }}$ & Search periapical granuloma & $\underline{859}$ & 04:08:45 \\
\hline$\# 2$ & $\underline{\text { Add }}$ & Search periapical abscess & $\underline{1760}$ & 04:08:28 \\
\hline$\underline{\# 1}$ & $\underline{\text { Add }}$ & Search periapical lesion & $\underline{1026}$ & 04:08:08 \\
\hline
\end{tabular}

\section{Inclusion Criteria}

Criteria for considering studies for this review

Types of Studies

1. Comparing both conventional Cone beam computed tomography and intra oral periapical radiography

\section{Types of Participants}

Patients of age greater than 16years having necrotic teeth and apical periodontitis.

\section{Types of Interventions}

Cone beam computed tomography and intra oral periapical radiography.

\section{Types of Outcome Measures}

Detection of periapical radiolucencies of both the method. 


\section{Exclusion Criteria}

The following studies were excluded,

- Case reports/case series

- Animal studies

- In vitro studies

\section{Chart 1: Search Flow Chart}

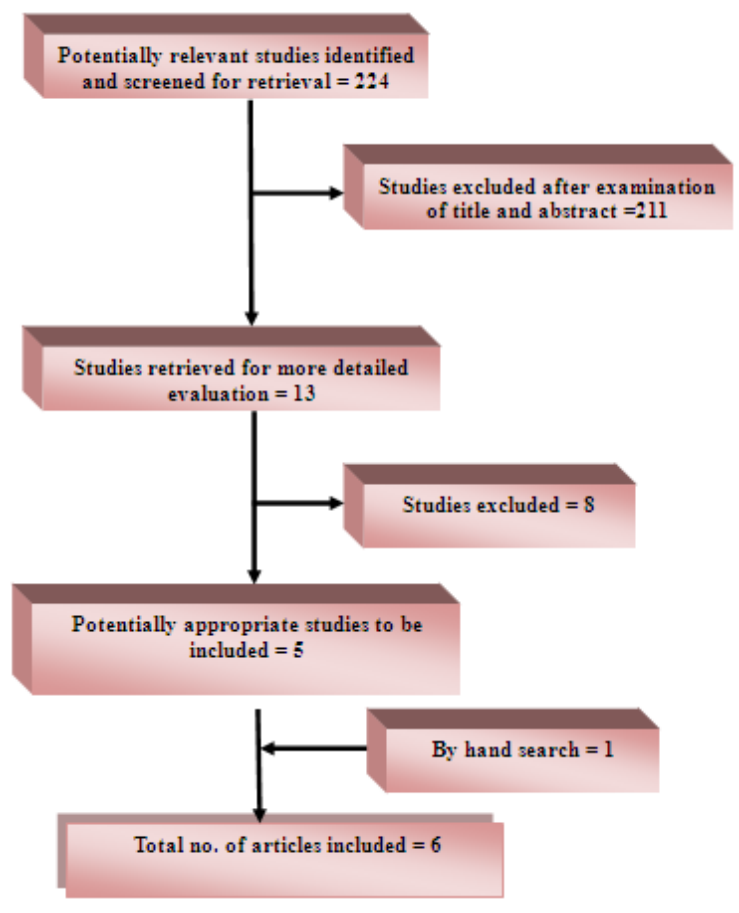

Table 1: Variables Of Interest

\begin{tabular}{|l|l|}
\hline S. No & Variables Of Interest \\
\hline 1. & Detection of periapical lesion \\
\hline
\end{tabular}

Table 2: Characteristics Of Excluded Studies

\begin{tabular}{|l|l|l|l|}
\hline S. No & Author & Year & Reason for Exclusion \\
\hline 1. & ${\text { Abella et } \mathrm{al}^{15}}^{15}$ & 2013 & CBCT compared with digial radiography \\
\hline 2. & ${\text { Balasundaram et } \mathrm{a}^{17}}^{17}$ & 2012 & $\begin{array}{l}\text { Only extent of lesion determined not the detection of } \\
\text { lesion }\end{array}$ \\
\hline 3. & Tsai P et al $^{18}$ & 2012 & In vitro \\
\hline 4. & Abella et al $^{16}$ & 2012 & Age group not satisfied \\
\hline 5. & Lennon S et al $^{20}$ & 2011 & $\begin{array}{l}\text { Only CBCT with different angles done not compared with } \\
\text { periapical radiograph }\end{array}$ \\
\hline 6. & ${\text { Paula }-\mathrm{Silva}^{2} \mathrm{al}^{8}}^{8}$ & 2009 & Animal study \\
\hline 7. & Patel S et al $^{11}$ & 2009 & In vitro \\
\hline 8. & Jorge et al $^{6}$ & 2008 & Animal study \\
\hline 9. & Velvart et al $^{13}$ & 2001 & Study done with CT not CBCT \\
\hline
\end{tabular}

\section{Results}

\section{Description of Studies}

The search identified 224 publications out of which 211 were excluded after reviewing the title or abstract. Full articles were obtained for 13 studies, 8 of these publications were excluded after reading the full text article, 1 article is obtained by hand search therefore a total of 6 publications fulfilled all criteria for inclusion.

Table 3: General Information Of Selected Articles 
Comparison of cone beam computed tomography and intra oral periapical radiography in ....

\begin{tabular}{|c|c|c|c|c|c|c|c|c|}
\hline $\begin{array}{l}\text { S. } \\
\text { No }\end{array}$ & Author & $\begin{array}{l}\text { Yea } \\
\mathbf{r}\end{array}$ & Country & $\begin{array}{l}\text { Study } \\
\text { Design }\end{array}$ & $\begin{array}{l}\text { Sampl } \\
\text { e Size }\end{array}$ & Age & Set-up & $\begin{array}{l}\text { Diagnostic method } \\
\text { used }\end{array}$ \\
\hline 1. & Sara lofthag et al ${ }^{5}$ & 2007 & Sweden & Clinical trial & 124 & $>16 y r s$ & University & $\begin{array}{l}\text { Cone beam } \\
\text { computed } \\
\text { tomography and } \\
\text { intra oral periapical } \\
\text { radiography. }\end{array}$ \\
\hline 2. & Kenneth M.T et al ${ }^{11}$ & 2008 & Singapore & Clinical trial & 156 & $>31 \mathrm{yrs}$ & University & $\begin{array}{l}\text { Cone beam } \\
\text { computed } \\
\text { tomography and } \\
\text { intra oral periapical } \\
\text { radiography. }\end{array}$ \\
\hline 3. & Estrela et $\mathrm{al}^{12}$ & 2008 & Brazil & Clinical trial & 596 & $>37$ & University & $\begin{array}{l}\text { Cone beam } \\
\text { computed } \\
\text { tomography and } \\
\text { intra oral periapical } \\
\text { radiography. }\end{array}$ \\
\hline 4. & Estrela et $\mathrm{al}^{7}$ & 2008 & Brazil & Clinical trial & 1508 & $>38$ & University & $\begin{array}{l}\text { Cone beam } \\
\text { computed } \\
\text { tomography and } \\
\text { intra oral periapical } \\
\text { radiography. }\end{array}$ \\
\hline 5. & S.patel et al ${ }^{13}$ & 2011 & $\begin{array}{l}\text { United } \\
\text { Kingdom }\end{array}$ & Clinical trial & 151 & $>18 \mathrm{yrs}$ & University & $\begin{array}{l}\text { Cone beam } \\
\text { computed } \\
\text { tomography and } \\
\text { intra oral periapical } \\
\text { radiography. }\end{array}$ \\
\hline 6. & Rafael Fernandez et al ${ }^{9}$ & 2013 & Colombia & Clinical trial & 208 & $>18 \mathrm{yrs}$ & $\begin{array}{l}\text { Clinical } \\
\text { trial }\end{array}$ & $\begin{array}{l}\text { Cone beam } \\
\text { computed } \\
\text { tomography and } \\
\text { intra oral periapical } \\
\text { radiography. }\end{array}$ \\
\hline
\end{tabular}

Table 4: Results

\begin{tabular}{|c|c|c|c|c|}
\hline $\begin{array}{c}\text { S. } \\
\text { No }\end{array}$ & Author and Year & Diagnostic Method Used & Results & Outcome \\
\hline 1. & $\begin{array}{l}\text { Sara Lofthag et al } \\
2007^{5}\end{array}$ & $\begin{array}{l}\text { Cone beam computed tomography } \\
\text { and intra oral periapical radiography. }\end{array}$ & $\begin{array}{l}\text { Intra oral periapical } \\
\text { radiograph- } 69.56 \% \\
\text { Cone beam computed } \\
\text { tomography }-91.3 \%\end{array}$ & $\begin{array}{l}\text { Cone beam computed } \\
\text { tomography is superior } \\
\text { to intra oral periapical } \\
\text { radiography. }\end{array}$ \\
\hline 2. & $\begin{array}{l}\text { Kenneth M T et } \\
\mathrm{al}^{11} \\
2008\end{array}$ & $\begin{array}{l}\text { Cone beam computed tomography } \\
\text { and intra oral periapical radiography. }\end{array}$ & $\begin{array}{l}\text { Intra oral periapical radiograph } \\
-46.1 \% \\
\text { Cone beam computed } \\
\text { tomography }-69.8 \%\end{array}$ & $\begin{array}{l}\text { Cone beam computed } \\
\text { tomography is superior } \\
\text { to intra oral periapical } \\
\text { radiography. }\end{array}$ \\
\hline 3. & Estrela et al $2008^{12}$ & $\begin{array}{l}\text { Cone beam computed tomography } \\
\text { and intra oral periapical radiography. }\end{array}$ & $\begin{array}{l}\text { Intra oral periapical radiograph } \\
-39.5 \% \\
\text { Cone beam computed } \\
\text { tomography }-60.9 \%\end{array}$ & $\begin{array}{l}\text { Cone beam computed } \\
\text { tomography is superior } \\
\text { to intra oral periapical } \\
\text { radiography. }\end{array}$ \\
\hline 4. & Estrela et al $2008^{7}$ & $\begin{array}{l}\text { Cone beam computed tomography } \\
\text { and intra oral periapical radiography. }\end{array}$ & $\begin{array}{l}\text { Intra oral periapical radiograph } \\
-35.3 \% \\
\text { Cone beam computed } \\
\text { tomography }-63.9 \%\end{array}$ & $\begin{array}{l}\text { Cone beam computed } \\
\text { tomography is superior } \\
\text { to intra oral periapical } \\
\text { radiography. }\end{array}$ \\
\hline 6. & $\begin{array}{l}\text { Rafael Fernandez } \\
\text { et al } 2013\end{array}$ & $\begin{array}{l}\text { Cone beam computed tomography } \\
\text { and intra oral periapical radiography. }\end{array}$ & $\begin{array}{l}\text { Intra oral periapical radiograph } \\
-5.7 \% \\
\text { Cone beam computed } \\
\text { tomography }-18.7 \%\end{array}$ & $\begin{array}{l}\text { Cone beam computed } \\
\text { tomography is superior to } \\
\text { intra oral periapical } \\
\text { radiography. }\end{array}$ \\
\hline
\end{tabular}




\section{Quality Assessment}

The quality assessment of included trials was undertaken independently as a part of data extraction process. Four main quality criteria were examined:

1. Method of Randomization, recorded as

a. Yes - Adequate as described in the text

b. No - Inadequate as described in the text

c. Unclear in the text

2. Allocation Concealment, recorded as

a. Yes - Adequate as described in the text

b. No - Inadequate as described in the text

c. Unclear in the text

3. Outcomes assessors blinded to intervention, recorded as

a. Yes - Adequate as described in the text

b. No - Inadequate as described in the text

c. Unclear in the text

4. Completeness of follow-up (was there a clear explanation for withdrawals and dropouts in each treatment group) assessed as:

a. Yes-Dropouts were explained

b. No-Dropouts were not explained

c. None -No Dropouts or withdrawals

Other methodological criteria examined included:

1. Presence or absence of sample size calculation

2. Comparability of groups at the start

3. Clear inclusion/ exclusion criteria

Presence/ absence of estimate of measurement error. The validity and reproducibility of the method of assessment.

Table 5: Evidence Of Selected Articles

\begin{tabular}{|l|l|l|l|l|}
\hline \multicolumn{1}{|c|}{ S. No } & \multicolumn{1}{|c|}{ Author } & \multicolumn{1}{|c|}{ Year } & \multicolumn{1}{c|}{ Study Design } & Level of Evidence \\
\hline 1. & Sara lofthag et al & 2007 & Clinical Trial & Level 3 \\
\hline 2. & Kenneth M T et al & 2008 & Clinical Trial & Level 3 \\
\hline 3. & Estrela et al & 2008 & Clinical Trial & Level 3 \\
\hline 4. & Estrela et al & 2008 & Clinical Trial & Level 3 \\
\hline 5. & S Patel et al & 2011 & Clinical Trial & Level 3 \\
\hline 6. & Rafael Fernandez et al & 2013 & Clinical Trial & Level 3 \\
\hline
\end{tabular}

Graph 1: Number Of Studies

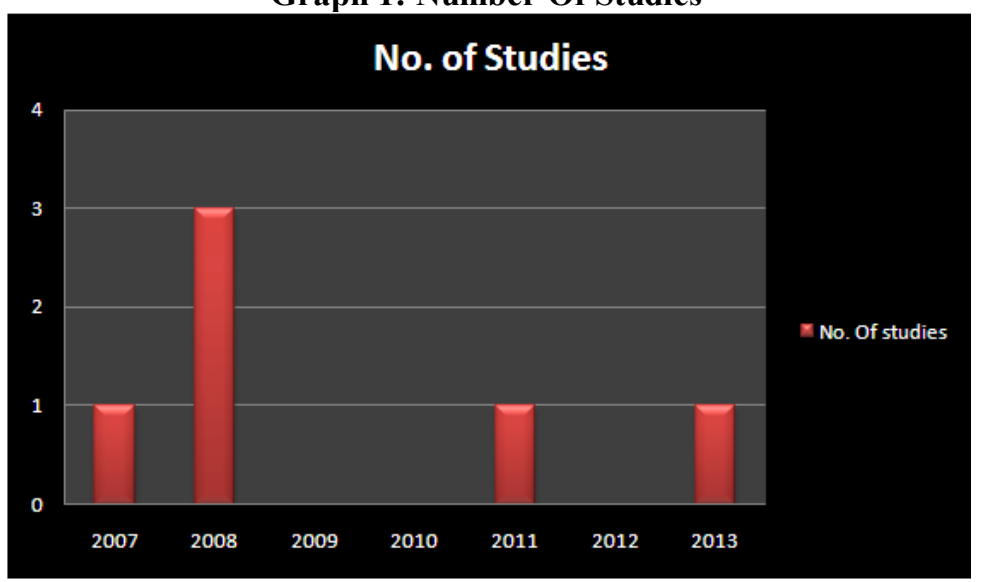




\section{Risk of Bias in Included Studies}

The assessments for the four main methodological quality items are shown in table. The study was assessed to have a "High risk" of bias if it did not record a "Yes" in three or more of the four main categories, "Moderate" if two out of four categories did not record a "Yes" and "Low" if randomization assessor blinding and completeness of follow - up were considered adequate.

Table 6: Risk Of Bias - Major Criteria

\begin{tabular}{|l|l|l|l|l|c|}
\hline \multicolumn{1}{|c|}{ Study } & \multicolumn{1}{|c|}{ Randomization } & \multicolumn{1}{c|}{$\begin{array}{c}\text { Allocation } \\
\text { Concealed }\end{array}$} & $\begin{array}{c}\text { Assessor } \\
\text { Blinding }\end{array}$ & Dropouts Described & Risk of Bias \\
\hline $\begin{array}{l}\text { Sara Lofthag et al } \\
2007\end{array}$ & No & No & No & None & High \\
\hline $\begin{array}{l}\text { Kenneth M T et al } \\
2008\end{array}$ & No & No & No & None & High \\
\hline $\begin{array}{l}\text { Estrela et al } \\
2008\end{array}$ & No & No & Yes & None & High \\
\hline $\begin{array}{l}\text { Estrela et al } \\
2008\end{array}$ & No & No & No & None & High \\
\hline $\begin{array}{l}\text { S Patel et al } \\
2011\end{array}$ & No & No & No & None & High \\
\hline $\begin{array}{l}\text { Rafael Fernandez et al } \\
2013\end{array}$ & No & No & No & None & High \\
\hline
\end{tabular}

Table 7: Risk Of Bias - Minor Criteria

\begin{tabular}{|l|l|l|l|l|}
\hline \multicolumn{1}{|c|}{ Study } & Sample Justified & Baseline Comparison & I/ E Criteria & Method Error \\
\hline $\begin{array}{l}\text { Sara Lofthag et al } \\
2007\end{array}$ & No & Yes & Yes & No \\
\hline $\begin{array}{l}\text { Kenneth M T et al } \\
2008\end{array}$ & No & Yes & Yes & No \\
\hline Estrela et al 2008 & No & Yes & Yes & No \\
\hline Estrela et al 2008 & No & Yes & Yes & No \\
\hline S Patel et al 2011 & No & Yes & Yes & No \\
\hline $\begin{array}{l}\text { Abella et al 2012 } \\
\text { Rafael Fernandez et al }\end{array}$ & No & Yes & Yes & No \\
\hline
\end{tabular}

\section{Interpretation of Results}

\section{Discussion}

First trial Saralfthang-Hansen et $\mathbf{a l}^{5}$ (2007), in a clinical trial analyzed 46 teeth out of which 5 had a clinical diagnosis of apical periodontitis 41 were endodontically treated, of which 23 had a post in one or more root canals. Among 46 teeth $32(69.5 \%)$ teeth were indentified with periapical lesion in intra oral periapical radiography radiograph and $42(91.3 \%)$ teeth were identified with periapical lesion in cone beam computed tomography

Second trial Kenneth M T et $\mathbf{a l}^{\mathbf{1 1}}$ (2008), in a clinical trial, analyzed 74 teeth yielding 156 roots with clinical signs of apical periodontitis. Among 156 roots $72(46.1 \%)$ teeth were indentified with periapical lesion in intra oral periapical radiography radiograph and $109(69.8 \%)$ teeth were identified with periapical lesion in cone beam computed tomography

Third trial Carols Estela et al $^{12}$ (2008), in a clinical trial, analyzed 1014 teeth with clinical signs of apical periodontitis. Among 1014 teeth 401(39.5\%) teeth were indentified with periapical lesion in intra oral periapical radiography radiograph and $618(60.9 \%)$ teeth were identified with periapical lesion in cone beam computed tomography

Fourth trial Carols Estela et $\mathbf{~ a l}^{7}$ (2008) in a clinical trial, analyzed 1508 teeth with clinical signs of apical periodontitis. Among 1508 teeth, $533(35.3 \%)$ teeth were indentified with periapical lesion in intra oral periapical radiography radiograph and $964(63.9 \%)$ teeth were identified with periapical lesion in cone beam computed tomography

Fifth trial S Patel et al ${ }^{13}$ (2011) in a clinical trial, analyzed 132 teeth (208) roots which is endodontic ally treated. Among 273 teeth, $55(20 \%)$ teeth were indentified with periapical lesion in intra oral periapical radiography radiograph and $130(48 \%)$ teeth were identified with periapical lesion in cone beam computed tomography 
Sixth clinical trial by Rafael et $\mathbf{a l}^{\mathbf{9}}, 2013$, in a clinical trial, analyzed roots with clinical signs of apical periodontitis. Among teeth, $12(5.7 \%) \%$ teeth were indentified with periapical lesion in intra oral periapical radiography and $39(18.7 \%)$ teeth were identified with periapical lesion in cone beam computed tomography

Six clinical trials compared the accuracy of cone beam computed tomography and intra oral periapical radiography in determining periapical lesion all the studies states that cone beam computed tomography is superior to intra oral periapical radiography in detection of periapical lesion with significant difference

\section{Defending the Results}

Ex vivo studies in which the detection of simulated periapical lesions has been assessed with cone beam computed tomography images and intraoral radiographs have all confirmed the superior diagnostic ability of cone beam computed tomography images over intraoral periapical radiographs (Stavropoulos and Wenzel 2007 , Ozen et al 2009, Patel et al 2009, Sogur et al2009) ${ }^{10}$. These findings have been reinforced by more recent in vivo dog studies

(Paula-Silva ${ }^{8}$ et al 2009). Intentionally created periapical lesions were induced around the roots of dog's teeth (one group had vital pulps to serve as a positive control). After 180 days (another group was left untreated to serve a negative control), intraoral radiographs and cone beam computed tomography scans were taken after which the animals were sacrificed, and the root apices and surrounding periapical tissues were evaluated histologically . CBCT helps in diagnosis of lesions extending lingually behind the tooth structure whereas IOPA cannot as it is a two dimensional image. These studies confirmed that cone beam computed tomography not only was more sensitive at detecting periapical lesions, but also had a higher overall accuracy when compared with intraoral periapical radiographs.

\section{Implications for Practice}

\section{Inference}

There is enough evidence that cone beam computed tomography is superior to intra oral periapical radiography in detection of periapical lesion with the only disadvantage being radiation exposure.

\section{Implications for Research}

Since there is adequate studies stating that cone beam computed tomography is superior to intra oral periapical radiography in detection of periapical lesion further research can be done by comparing cone beam computed tomography with other methods such as ultrasound etc.,

\section{Report of Outlier Data}

No outlier data obtained.

\section{Summary}

The aim of this systematic review was to compare accuracy of cone beam computed tomography $(\mathrm{CBCT})$ and intra oral periapical radiography on diagnosis of periapical pathology

Trials were selected if they met the following criteria. Clinical trials comparing cone beam computed tomography with conventional intra oral periapical radiography

The databases PUBMED CENTRAL and MEDLINE were searched for the related topic from 1996 till July 2013. The search identified 224 publications out of which 211 were excluded after reviewing the title or abstract. Full articles were obtained for 13 studies, 8 of these publications were excluded after reading the full text article, 1 article is obtained by hand search therefore a total of 6 publications fulfilled all criteria for inclusion

\section{Conclusion}

There is enough evidence that cone beam computed tomography is superior to intraoral periapical radiography in detection of periapical lesion with the only disadvantage being radiation exposure

\section{References}

[1]. Feldkamp LA, Davis LC, Kress JW. Practical cone-beam algorithm. J Opt Soc Am 1994: 1: 612-619.

[2]. Arai Y, Tammisalo E, Iwai K, Hashimoto K, Shinoda K. Development of a compact computed tomographic apparatus for dental use. Dentomaxillofac Radiol 1999: 28: 245-248.

[3]. Simon JHS. Incidence of periapical cysts in relation to the root canal. J Endod 1980;6:845-8.

[4]. Kaffe I, Gratt BM. Variations in the radiographic interpretation of the periapical dental region. J Endod 1988;14:330.

[5]. Lofthag-Hansen S, Hummonen S, Gröndahl K, Gröndahl H-G. Limited cone-beam CT and intraoral radiography for the diagnosis of periapical pathology. Oral Surg Oral Med Oral Pathol Oral Radiol Endod 2007;103:114 -9.

[6]. Jorge EG, Tanomaru-Filho M, Gonçalves M, Tanomaru JM. Detection of periapical lesion development by conventional radiography or computed tomography. Oral Surg Oral Med Oral Pathol Oral Radiol Endod. 2008 Jul;106(1):e56-61 
[7]. Estrela C, Bueno MR, Leles CR, Azevedo B, Azevedo JR. Accuracy of cone beam computed tomography and panoramic and periapical radiography for detection of apical periodontitis.J Endod. 2008 Mar;34(3):273-9. doi: 10.1016/j.joen.2007.11.023.

[8]. De Paula-Silva FW, Wu MK, Leonardo MR, et al. Accuracy of periapical radiography and cone beam computed tomography in diagnosing apical periodontitis using histopathological findings as a gold standard. J Endod 2009;35:1009-12.

[9]. Rafael Fernandez, Diego Cadavid, Sandra M. Zapata,Luis G.Alvarez, Felipe A. Restrepo, Impact of three radiographic methods in the outcome of nonsurgical endodontic treatment: A Five-Year Follow-up. J Endod 2013;37:1-7.

[10]. Petersson A, Axelsson S, Davidson T, et al. Radiological diagnosis of periapical bone tissue lesions in endodontics: a systematic review. Int Endod J 2012;45:783-801.

[11]. KMT Low, Dula K, Bürgin W, von Arx T. Comparison of periapical radiography and limited cone-beam tomography in posterior maxillary teeth referred for apical surgery. J Endod. 2008 May;34(5):557-62. doi: 10.1016/j.joen.2008.02.022.

[12]. Estrela C, Bueno MR, Azevedo BC, et al. A new periapical index based on cone beam computed tomography. J Endod 2008;34:1325-31.

[13]. Velvart P, Hecker H, Tillinger G. Detection of the apical lesion and the mandibular canal in conventional radiography and computed tomography. Oral Surg Oral Med Oral Pathol Oral Radiol Endod 2001;92:682- 8 .

[14]. Estrela C, Leles CR, Hollanda ACB, Moura MS, Pécora JD. Prevalence and risk factors of apical periodontitis in endodontically treated teeth in a selected population of Brazilian adults. Braz Dent J 2013;19:34 -9.

[15]. Abella F, Patel S, Durán-Sindreu F, Mercadé M, Bueno R, Roig M. An evaluation of the periapical status of teeth with necrotic pulps using periapical radiography and cone-beam computed tomography.Int Endod J. 2013 Jul 3. doi: 10.1111/iej.12159.

[16]. Abella F, Patel S, Duran-Sindreu F, Mercadé M, Bueno R, Roig M. Evaluating the periapical status of teeth with irreversible pulpitis by using cone-beam computed tomography scanning and periapical radiographs. J Endod. 2012 Dec;38(12):1588-91. doi: J Endod .2012.09.003.

[17]. Balasundaram A, Shah P, Hoen MM, Wheater MA, Bringas JS, Gartner A, Geist JR. Comparison of cone-beam computed tomography and periapical radiography in predicting treatment decision for periapical lesions: a clinical study. Int $\mathrm{J}$ Dent. 2012;2012:920815. doi: 10.1155/2012/920815

[18]. Tsai P, Torabinejad M, Rice D, Azevedo B. Accuracy of cone-beam computed tomography and periapical radiography in detecting small periapical lesions. J Endod. 2012 Jul;38(7):965-70. doi: 10.1016/j.joen.2012.03.001. Epub 2012 May.

[19]. Patel S, Wilson R, Dawood A, Mannocci F The detection of periapical pathosis using periapical radiography and cone beam computed tomography - part 1: pre-operative status. Int Endod J. 2012 Aug;45(8):702-10. doi: 10.1111/j.1365-2591.2011.01989.x. Epub 2011 Dec 2.

[20]. Lennon S, Patel S, Foschi F, Wilson R, Davies J, Mannocci F. Diagnostic accuracy of limited-volume cone-beam computed tomography in the detection of periapical bone loss: $360^{\circ}$ scans versus $180^{\circ}$ scans. Int Endod J. 2011 Dec;44(12):1118-27.

[21]. Patel S, Dawood A, Mannocci F, Wilson R, Pitt Ford T. Detection of periapical bone defects in human jaws using cone beam computed tomography and intraoral radiography.Int Endod J. 2009 Jun;42(6):507-15. doi: 10.1111/j.1365-2591.2008.01538.x. Epub 2009 Mar 2.

[22]. Estrela C, Bueno MR, Azevedo BC, Azevedo JR, Pécora JD. A new periapical index based on cone beam computed tomography. J Endod. 2008 Nov;34(11):1325-31. doi: 10.1016/j.joen.2008.08.013. Epub 2008 Sep

[23]. Ørstavik D, Kerekes K, Eriksen HM. The periapical index: a scoring system for radiographic assessment of apical periodontitis. Endod Dent Traumatol 1986;2:20-34.

[24]. Velvart P, Hecker H, Tillinger G. Detection of the apical lesion and the mandibular canal in conventional radiography and computed tomography. Oral Surg Oral Med Oral Pathol Oral Radiol Endod. 2001 Dec;92(6):682-8. 